\title{
Unified Fixed Point Theorems via Common Limit Range Property in Modified Intuitionistic Fuzzy Metric Spaces
}

\author{
Mohammad Imdad, ${ }^{1}$ Sunny Chauhan, ${ }^{2}$ and Sumitra Dalal ${ }^{3}$ \\ ${ }^{1}$ Department of Mathematics, Aligarh Muslim University, Aligarh 202 002, India \\ ${ }^{2}$ Near Nehru Training Centre, H. No. 274, Nai Basti B-14, Bijnor, Uttar Pradesh 246701, India \\ ${ }^{3}$ Department of Mathematics, Jazan University, Saudi Arabia
}

Correspondence should be addressed to Sunny Chauhan; sun.gkv@gmail.com

Received 7 June 2013; Revised 1 September 2013; Accepted 8 September 2013

Academic Editor: E. Karapınar

Copyright (C) 2013 Mohammad Imdad et al. This is an open access article distributed under the Creative Commons Attribution License, which permits unrestricted use, distribution, and reproduction in any medium, provided the original work is properly cited.

\begin{abstract}
The purpose of this paper is to emphasize the role of "common limit range property" to ascertain the existence of common fixed points in modified intuitionistic fuzzy metric spaces enjoying an implicit function utilized in Tanveer et al. (2012) and Imdad et al. (2012). As an application to our main result, we derive a fixed point theorem for finite families of self-mappings. We also give some examples which demonstrate the validity of the hypotheses and degree of generality of our main results. Our results improve and extend several previously known fixed point theorems of the existing literature.
\end{abstract}

\section{Introduction}

The fruitful and productive idea of fuzzy set was initiated by Zadeh [1]. In an attempt to generalize the idea of fuzzy set, Atanassov [2] introduced the notion of intuitionistic fuzzy set. Thereafter, Çoker [3] defined a topology on intuitionistic fuzzy sets, while Mondal and Samanta [4] introduced the idea of intuitionistic gradation of openness. Park [5] introduced the notion of intuitionistic fuzzy metric space (abbreviated by IFMS in the sequel) as a generalization of fuzzy metric space, especially the one due to George and Veeramani [6]. In recent years, many authors proved a multitude of fixed point theorems in IFMS (e.g., see [7-15]).

Later on, Gregori et al. [16] showed that the topology induced by fuzzy metric coincides with the topology induced by intuitionistic fuzzy metric. In an attempt to remove this shortcoming, Saadati and Park [17] proposed the idea of modified IFMS wherein the notions of continuous t-norm and continuous $\mathrm{t}$-conorm are employed besides adopting the notion of compatible mappings (essentially due to Jungck [18]). Jain et al. [19] proved some unique common fixed point theorems for four self-mappings satisfying a new contractive condition in modified IFMS through compatibility of type $(P)$. Saadati and Park [17] extended the notion of weak compatibility (due to Jungck and Rhoades [20]) to modified IFMS. However, the study of common fixed points of noncompatible mappings due to Pant [21] is also equally natural. Tanveer et al. [22] and Imdad et al. [23] utilized the notions of the property (E.A) (due to Aamri and Moutawakil [24]) and the common property (E.A) (due to Liu et al. [25]) to prove some interesting results in modified intuitionistic fuzzy metric spaces. One may notice that the property (E.A) does require the closedness of certain underlying subspaces to ascertain the existence of common fixed point. Sintunavarat and Kumam [26] coined the idea of "common limit range property" which never requires the closedness of any underlying subspace for the existence of common fixed points (also see [27]). Most recently, Chauhan et al. [28, 29] and Sintunavarat et al. [14] proved some interesting fixed point results for mappings defined on modified IFMS via common limit range property. Imdad et al. [30] extended the notion of common limit range property to two pairs of self-mappings and proved some fixed point results in Menger and metric spaces. We cite some recent papers (e.g., [31-37]) which demonstrate the superiority of common limit range property over the property (E.A) in various settings. 
In this paper, utilizing an implicit function due to Tanveer et al. [22] (also Imdad et al. [23]), we prove some common fixed point theorems for two pairs of weakly compatible mappings in modified IFMS employing the common limit range property. In process, many known results (especially those contained in Imdad et al. [23]) are enriched and improved. Some related results are also derived besides furnishing illustrative examples.

\section{Preliminaries}

Lemma 1 (see [38]). Consider the set $L^{*}$ and operation $\leq_{L^{*}}$ defined by

$$
L^{*}=\left\{\left(x_{1}, x_{2}\right):\left(x_{1}, x_{2}\right) \in[0,1]^{2}, x_{1}+x_{2} \leq 1\right\},
$$

$\left(x_{1}, x_{2}\right) \leq_{L^{*}}\left(y_{1}, y_{2}\right) \Leftrightarrow x_{1} \leq y_{1}$ and $x_{2} \geq y_{2}$, for every $\left(x_{1}, x_{2}\right),\left(y_{1}, y_{2}\right) \in L^{*}$. Then $\left(L^{*}, \leq_{L^{*}}\right)$ is a complete lattice.

Definition 2 (see [2]). An intuitionistic fuzzy set $A_{\zeta, \eta}$ in a universe $\mathcal{U}$ is an object $A_{\zeta, \eta}=\left\{\left(\zeta_{A}(u), \eta_{A}(u) \mid u \in \mathscr{U}\right)\right\}$, where, for all $u \in \mathcal{U}, \zeta_{A}(u) \in[0,1]$ and $\eta_{A}(u) \in[0,1]$ are, respectively, called the membership degree and the nonmembership degree of $u \in A_{\zeta, \eta}$ which also satisfy $\zeta_{A}(u)+\eta_{A}(u) \leq 1$.

For every $z_{i}=\left(x_{i}, y_{i}\right) \in L^{*}$, if $c_{i} \in[0,1]$ such that $\sum_{j=1}^{n} c_{j}=1$, then it is easy to see that

$$
\begin{aligned}
c_{1}\left(x_{1}, y_{1}\right)+\cdots+c_{n}\left(x_{n}, y_{n}\right) & =\sum_{j=1}^{n} c_{j}\left(x_{j}, y_{j}\right) \\
& =\left(\sum_{j=1}^{n} c_{j} x_{j}, \sum_{j=1}^{n} c_{j} y_{j}\right) \in L^{*} .
\end{aligned}
$$

We denote its units by $0_{L^{*}}=(0,1)$ and $1_{L^{*}}=(1,0)$. Classically, a triangular norm $*=\mathrm{T}$ on $[0,1]$ is defined as an increasing, commutative, associative mapping $\mathrm{T}:[0,1]^{2} \rightarrow$ $[0,1]$ satisfying $\mathrm{T}(1, x)=1 * x=x$, for all $x \in[0,1]$. A triangular conorm $\mathrm{S}=\diamond$ is defined as an increasing, commutative, associative mapping $S:[0,1]^{2} \rightarrow[0,1]$ satisfying $\mathrm{S}(0, x)=0 \diamond x=x$, for all $x \in[0,1]$. Using the lattice $\left(L^{*}, \leq_{L^{*}}\right)$, these definitions can be easily extended.

Definition 3 (see [39]). A triangular norm (t-norm) on $L^{*}$ is a mapping $\mathscr{T}:\left(L^{*}\right)^{2} \rightarrow L^{*}$ satisfying the following conditions for all $x, y, x^{\prime}, y^{\prime} \in L^{*}$ :
(1) $\mathscr{T}\left(x, 1_{L^{*}}\right)=x$,
(2) $\mathscr{T}(x, y)=\mathscr{T}(y, x)$,
(3) $\mathscr{T}(x, \mathscr{T}(y, z))=\mathscr{T}(\mathscr{T}(x, y), z)$,
(4) $x \leq_{L^{*}} x^{\prime}$ and $y \leq_{L^{*}} y^{\prime} \Rightarrow \mathscr{T}(x, y) \leq_{L^{*}} \mathscr{T}\left(x^{\prime}, y^{\prime}\right)$.

Definition 4 (see $[38,39])$. A continuous t-norm $\mathscr{T}$ on $L^{*}$ is called continuous t-representable if and only if there exist a continuous t-norm $*$ and a continuous t-conorm $\diamond$ on $[0,1]$ such that, for all $x=\left(x_{1}, x_{2}\right), y=\left(y_{1}, y_{2}\right) \in L^{*}$,

$$
\mathscr{T}(x, y)=\left(x_{1} * y_{1}, x_{2} \diamond y_{2}\right) \text {. }
$$

Now, we define a sequence $\left\{\mathscr{T}_{n}\right\}$ recursively by $\left\{\mathscr{T}^{1}=\mathscr{T}\right\}$ and

$$
\begin{aligned}
\mathscr{T}^{n}\left(x^{(1)}, \ldots, x^{(n+1)}\right) \\
\quad=\mathscr{T}\left(\mathscr{T}^{n-1}\left(x^{(1)}, \ldots, x^{(n)}\right), x^{(n+1)}\right),
\end{aligned}
$$

for $n \geq 2$ and $x^{(i)} \in L^{*}$.

Definition 5 (see $[38,39])$. A negator on $L^{*}$ is any decreasing mapping $\mathscr{N}: L^{*} \rightarrow L^{*}$ satisfying $\mathcal{N}\left(0_{L^{*}}\right)=1_{L^{*}}$ and $\mathcal{N}\left(1_{L^{*}}\right)=0_{L^{*}}$. If $\mathcal{N}(\mathcal{N}(x))=x$, for all $x \in L^{*}$, then $\mathcal{N}$ is called an involutive negator. A negator on $[0,1]$ is a decreasing mapping $N:[0,1] \rightarrow[0,1]$ satisfying $N(0)=1$ and $N(1)=0$. Notice that $N_{s}$ stands for standard negator on $[0,1]$ defined by (for all $x \in[0,1]) N_{s}(x)=1-x$.

Definition 6 (see [17]). Let $M, N$ be fuzzy sets from $X^{2} \times$ $(0, \infty)$ to $[0,1]$ such that $M(x, y, t)+N(x, y, t) \leq 1$ for all $x, y \in X$ and $t>0$. The 3 -tuple $\left(X, \mathscr{M}_{M, N}, \mathscr{T}\right)$ is said to be a modified IFMS if $X$ is an arbitrary nonempty set, $\mathscr{T}$ is a continuous t-representable, and $\mathscr{M}_{M, N}$ is an intuitionistic fuzzy set from $X^{2} \times(0, \infty) \rightarrow L^{*}$ satisfying the following conditions (for every $x, y, z \in X$ and $t, s>0$ ):

(1) $\mathscr{M}_{M, N}(x, y, t)>_{L^{*}} 0_{L^{*}}$,

(2) $\mathscr{M}_{M, N}(x, y, t)=1_{L^{*}}$ if and only if $x=y$,

(3) $\mathscr{M}_{M, N}(x, y, t)=\mathscr{M}_{M, N}(y, x, t)$,

(4) $\mathscr{M}_{M, N}(x, y, t+s) \geq_{L^{*}} \mathscr{T}\left(\mathscr{M}_{M, N}(x, z, t), \mathscr{M}_{M, N}(z, y, s)\right)$,

(5) $\mathscr{M}_{M, N}(x, y, \cdot):(0, \infty) \rightarrow L^{*}$ is continuous.

In this case, $\mathscr{M}_{M, N}$ is called a modified intuitionistic fuzzy metric. Here,

$$
\mathscr{M}_{M, N}(x, y, t)=(M(x, y, t), N(x, y, t)) .
$$

Remark 7. In an intuitionistic fuzzy metric space $\left(X, \mathscr{M}_{M, N}\right.$, $\mathscr{T}), M(x, y, \cdot)$ is nondecreasing and $N(x, y, \cdot)$ is nonincreasing for all $x, y \in X$. Hence $\left(X, \mathscr{M}_{M, N}, \mathscr{T}\right)$ is nondecreasing function for all $x, y \in X$.

Example 8 (see [17]). Let $(X, d)$ be a metric space. Define $\mathscr{T}(a, b)=\left(a_{1} b_{1}, \min \left\{a_{2}+b_{2}, 1\right\}\right)$ for all $a=\left(a_{1}, a_{2}\right)$ and $b=\left(b_{1}, b_{2}\right) \in L^{*}$, and let $M$ and $N$ be fuzzy sets on $X^{2} \times(0, \infty)$ defined as follows:

$$
\begin{aligned}
\mathscr{M}_{M, N}(x, y, t) & =(M(x, y, t), N(x, y, t)) \\
& =\left(\frac{h t^{n}}{h t^{n}+m d(x, y)}, \frac{m d(x, y)}{h t^{n}+m d(x, y)}\right),
\end{aligned}
$$

for all $h, m, n, t \in \mathbb{R}^{+}$. Then $\left(X, \mathscr{M}_{M, N}, \mathscr{T}\right)$ is a modified IFMS. Example 9 (see [17]). Let $X=\mathbb{N}$. Define $\mathscr{T}(a, b)=$ $\left(\max \left\{0, a_{1}+b_{1}-1\right\}, a_{2}+b_{2}-a_{2} b_{2}\right)$ for all $a=\left(a_{1}, a_{2}\right)$ and $b=\left(b_{1}, b_{2}\right) \in L^{*}$, and let $M$ and $N$ be fuzzy sets on $X^{2} \times(0, \infty)$. 
Then $\mathscr{M}_{M, N}(x, y, t)$ is defined as (for all $x, y \in X$ and $t>0$ ) follows:

$$
\begin{aligned}
\mathscr{M}_{M, N}(x, y, t)= & (M(x, y, t), N(x, y, t)) \\
& = \begin{cases}\left(\frac{x}{y}, \frac{y-x}{y}\right), & \text { if } x \leq y, \\
\left(\frac{y}{x}, \frac{x-y}{x}\right), & \text { if } y \leq x .\end{cases}
\end{aligned}
$$

Then $\left(X, \mathscr{M}_{M, N}, \mathscr{T}\right)$ is a modified IFMS.

Definition 10 (see [17]). Let $\left(X, \mathscr{M}_{M, N}, \mathscr{T}\right)$ be a modified IFMS. For $t>0$, define the open ball $\mathscr{B}(x, r, t)$ with center $x \in X$ and radius $0<r<1$ as

$$
\mathscr{B}(x, r, t)=\left\{y \in X: \mathscr{M}_{M, N}(x, y, t)>_{L^{*}}\left(N_{s}(r), r\right)\right\} .
$$

A subset $\mathscr{A} \subset X$ is called open if for each $x \in \mathscr{A}$ there exist $t>0$ and $0<r<1$ such that $\mathscr{B}(x, r, t) \subseteq \mathscr{A}$. If $\tau_{\mathscr{M}_{M, N}}$ denotes the family of all open subsets of $X$, then $\tau_{\mathscr{M}_{M, N}}$ is called the topology induced by intuitionistic fuzzy metric $\mathscr{M}_{M, N}$. Notice that this topology is Hausdorff (see [5], Remark 3.3, Theorem 3.5).

Definition 11 (see [17]). A sequence $\left\{x_{n}\right\}$ in a modified IFMS $\left(X, \mathscr{M}_{M, N}, \mathscr{T}\right)$ is called a Cauchy sequence if for each $0<\epsilon<$ 1 and $t>0$ there exists $n_{0} \in \mathbb{N}$ such that

$$
\mathscr{M}_{M, N}\left(x_{n}, y_{m}, t\right)>_{L^{*}}\left(N_{s}(\epsilon), \epsilon\right),
$$

and for each $n, m \geq n_{0}$, where $N_{s}$ is a standard negator. The sequence $\left\{x_{n}\right\}$ is said to be convergent to $x \in X$ in the modified IFMS $\left(X, \mathscr{M}_{M, N}, \mathscr{T}\right)$ and is generally denoted by $x_{n} \rightarrow \mathscr{M}_{M, N} x$ if $\mathscr{M}_{M, N}\left(x_{n}, x, t\right) \rightarrow 1_{L^{*}}$ whenever $n \rightarrow \infty$ for every $t>0$. A modified IFMS is said to be complete if and only if every Cauchy sequence is convergent.

Lemma 12 (see [17]). Let $\mathscr{M}_{M, N}$ be an intuitionistic fuzzy metric. Then, for any $t>0, M_{M, N}(x, y, t)$ is nondecreasing with respect to $t$ in $\left(L^{*}, \leq_{L^{*}}\right)$, for all $x, y \in X$.

Definition 13 (see [17]). Let $\left(X, \mathscr{M}_{M, N}, \mathscr{T}\right)$ be a modified IFMS. Then $\mathscr{M}_{M, N}$ is said to be continuous on $X \times X \times(0, \infty)$, if

$$
\lim _{n \rightarrow \infty} \mathscr{M}_{M, N}\left(x_{n}, y_{n}, t_{n}\right)=\mathscr{M}_{M, N}(x, y, t)
$$

whenever a sequence $\left\{\left(x_{n}, y_{n}, t_{n}\right)\right\}$ in $X \times X \times(0, \infty)$ converges to a point $\{(x, y, t)\} \in X \times X \times(0, \infty)$; that is,

$$
\begin{gathered}
\lim _{n \rightarrow \infty} \mathscr{M}_{M, N}\left(x_{n}, x, t\right)=\lim _{n \rightarrow \infty} \mathscr{M}_{M, N}(y, y, t)=1_{L^{*}}, \\
\lim _{n \rightarrow \infty} \mathscr{M}_{M, N}\left(x, y, t_{n}\right)=\mathscr{M}_{M, N}(x, y, t) .
\end{gathered}
$$

Lemma 14 (see [17]). Let (X, $\left.\mathscr{M}_{M, N}, \mathscr{T}\right)$ be a modified IFMS. Then, $\mathscr{M}_{M, N}$ is continuous function on $X \times X \times(0, \infty)$.

Definition 15. Let $A$ and $S$ be two mappings from a modified IFM space $\left(X, \mathscr{M}_{M, N}, \mathscr{T}\right)$ into itself. Then this pair of mappings is said to be

(1) commuting if $A S x=S A x$, for all $x \in X$;
(2) weakly commuting [17] if

$$
\mathscr{M}_{M, N}(A S x, S A x, t) \geq_{L^{*}} \mathscr{M}_{M, N}(A x, S x, t),
$$

for all $x \in X$ and $t>0$;

(3) compatible [17] if

$$
\lim _{n \rightarrow \infty} \mathscr{M}_{M, N}\left(A S x_{n}, S A x_{n}, t\right)=1_{L^{*}}
$$

for all $t>0$ whenever $\left\{x_{n}\right\}$ is a sequence in $X$ such that

$$
\lim _{n \rightarrow \infty} A x_{n}=\lim _{n \rightarrow \infty} S x_{n}=x \in X
$$

(4) noncompatible [22] if there exists at least one sequence $\left\{x_{n}\right\}$ in $X$ such that

$$
\lim _{n \rightarrow \infty} A x_{n}=\lim _{n \rightarrow \infty} S x_{n}=x \in X,
$$

but $\lim _{n \rightarrow \infty} \mathscr{M}_{M, N}\left(A S x_{n}, S A x_{n}, t\right) \neq 1_{L^{*}}$ or nonexistent for at least one $t>0$.

Definition 16 (see [40]). Two families of self-mappings $\left\{A_{i}\right\}_{i=1}^{m}$ and $\left\{S_{k}\right\}_{k=1}^{n}$ are said to be pairwise commuting if

(1) $A_{a} A_{b}=A_{b} A_{a}$, for all $a, b \in\{1,2, \ldots, m\}$;

(2) $S_{c} S_{d}=S_{d} S_{c}$, for all $c, d \in\{1,2, \ldots, n\}$;

(3) $A_{a} S_{c}=S_{c} A_{a}$, for all $a \in\{1,2, \ldots, m\}$ and $c \in$ $\{1,2, \ldots, n\}$.

Definition 17 (see [12]). Let $A$ and $S$ be two mappings from a modified IFMS $\left(X, \mathscr{M}_{M, N}, \mathscr{T}\right)$ into itself. Then this pair of mappings is said to satisfy the property (E.A) if there exists a sequence $\left\{x_{n}\right\}$ in $X$ such that for all $t>0$

$$
\begin{aligned}
\lim _{n \rightarrow \infty} & \mathscr{M}_{M, N}\left(A x_{n}, z, t\right) \\
= & \lim _{n \rightarrow \infty} \mathscr{M}_{M, N}\left(S x_{n}, z, t\right)=1_{L^{*}},
\end{aligned}
$$

for some $z \in X$.

Definition 18 (see [22]). Two pairs $(A, S)$ and $(B, T)$ of selfmappings of a modified IFMS $\left(X, \mathscr{M}_{M, N}, \mathscr{T}\right)$ are said to satisfy the common property (E.A) if there exist sequences $\left\{x_{n}\right\}$ and $\left\{y_{n}\right\}$ in $X$ such that

$$
\begin{aligned}
\lim _{n \rightarrow \infty} \mathscr{M}_{M, N}\left(A x_{n}, z, t\right) & =\lim _{n \rightarrow \infty} \mathscr{M}_{M, N}\left(S x_{n}, z, t\right) \\
& =\lim _{n \rightarrow \infty} \mathscr{M}_{M, N}\left(B y_{n}, z, t\right) \\
& =\lim _{n \rightarrow \infty} \mathscr{M}_{M, N}\left(T y_{n}, z, t\right)=1_{L^{*}},
\end{aligned}
$$

for some $z \in X$ and $t>0$.

Definition 19 (see [14]). A pair $(A, S)$ of self-mappings of a modified IFMS $\left(X, \mathscr{M}_{M, N}, \mathscr{T}\right)$ is said to satisfy the common limit range property with respect to $S$, denoted by $\left(\mathrm{CLR}_{S}\right)$, if there exists a sequence $\left\{x_{n}\right\}$ in $X$ such that for all $t>0$

$$
\lim _{n \rightarrow \infty} \mathscr{M}_{M, N}\left(A x_{n}, z, t\right)=\lim _{n \rightarrow \infty} \mathscr{M}_{M, N}\left(S x_{n}, z, t\right)=1_{L^{*}},
$$

where $z \in S(X)$.

Thus, one can infer that a pair $(A, S)$ satisfying the property (E.A) along with closedness of the subspace $S(X)$ always 
enjoys the $\left(C L R_{S}\right)$ property with respect to the mapping $S$ (see $[14,29])$.

Now, we extend common limit range property for two pairs of self-mappings in the framework of modified IFMS $\left(X, \mathscr{M}_{M, N}, \mathscr{T}\right)$ as follows.

Definition 20. Two pairs $(A, S)$ and $(B, T)$ of self-mappings of a modified IFMS $\left(X, \mathscr{M}_{M, N}, \mathscr{T}\right)$ are said to satisfy the common limit range property with respect to mappings $S$ and $T$, denoted by $\left(\mathrm{CLR}_{S T}\right)$, if there exist two sequences $\left\{x_{n}\right\}$ and $\left\{y_{n}\right\}$ in $X$ such that

$$
\begin{aligned}
\lim _{n \rightarrow \infty} \mathscr{M}_{M, N}\left(A x_{n}, z, t\right) & =\lim _{n \rightarrow \infty} \mathscr{M}_{M, N}\left(S x_{n}, z, t\right) \\
& =\lim _{n \rightarrow \infty} \mathscr{M}_{M, N}\left(B y_{n}, z, t\right) \\
& =\lim _{n \rightarrow \infty} \mathscr{M}_{M, N}\left(T y_{n}, z, t\right)=1_{L^{*}},
\end{aligned}
$$

where $z \in S(X) \cap T(X)$ and $t>0$.

By setting $A=B$ and $S=T$ in Definition 20 implies Definition 19 (due to Sintunavarat et al. [14]), whereas Definition 20 implies Definition 18, but the converse implications are not true in general. The following example substantiates this fact.

Example 21. Let $\left(X, \mathscr{M}_{M, N}, \mathscr{T}\right)$ be a modified IFMS, where $X=[3,20]$ and $\mathscr{M}_{M, N}(x, y, t)=(t /(t+|x-y|),|x-y| /(t+$ $|x-y|))$ for all $x, y \in X$ and $t>0$. Define four self-mappings $A, B, S$, and $T$ on $X$ as

$$
\begin{aligned}
& A(x)= \begin{cases}7, & \text { if } x=3 ; \\
\frac{x+1}{5}, & \text { if } 3<x \leq 14 ;\end{cases} \\
& B(x)= \begin{cases}\frac{4,}{4 x+3} & \text { if } x>14, \\
13, & \text { if } 3<x \leq 14 ;\end{cases} \\
& S(x)= \begin{cases}5, & \text { if } x>14, \\
\frac{15,}{2 x-1)}, & \text { if } x>3 ; \\
\frac{2}{9}, & \text { if } 3<x \leq 14 ;\end{cases} \\
& T(x)= \begin{cases}\frac{6,}{x+3} & \text { if } x=3 ; \\
\frac{17,}{2} & \text { if } 3<x \leq 14 ;\end{cases}
\end{aligned}
$$

If we choose two sequences as $\left\{x_{n}\right\}=\{14+1 / n\}_{n \in \mathbb{N}}$ and $\left.\left\{y_{n}\right\}=\{3+1 / n\}_{n \in \mathbb{N}}\right)$, then the pairs $(A, S)$ and $(B, T)$ enjoy the common property (E.A) for all $t>0$ :

$$
\begin{aligned}
\lim _{n \rightarrow \infty} \mathscr{M}_{M, N}\left(A x_{n}, 3, t\right) & =\lim _{n \rightarrow \infty} \mathscr{M}_{M, N}\left(S x_{n}, 3, t\right) \\
& =\lim _{n \rightarrow \infty} \mathscr{M}_{M, N}\left(B y_{n}, 3, t\right) \\
& =\lim _{n \rightarrow \infty} \mathscr{M}_{M, N}\left(T y_{n}, 3, t\right)=1_{L^{*}},
\end{aligned}
$$

where $3 \in X$. Here it is noticed that $3 \notin S(X) \cap T(X)$. Therefore, the pairs $(A, S)$ and $(B, T)$ do not satisfy the common limit range property with respect to mappings $S$ and $T$.

In view of Example 21, the following proposition is predictable.

Proposition 22. If the pairs $(A, S)$ and $(B, T)$ share the common property (E.A) and $S(X)$ as well as $T(X)$ are closed subsets of $X$, then the pairs also enjoy the $\left(C L R_{S T}\right)$ property.

\section{Implicit Relations}

On the lines of Imdad et al. [23], we adopt an implicit function which covers a multitude of contraction conditions in one go as exhibited by demonstrative examples.

Let $\Psi$ be the set of all upper continuous functions $\varphi\left(t_{1}, t_{2}, t_{3}, t_{4}, t_{5}, t_{6}\right): L^{*^{6}} \rightarrow L^{*}$, satisfying the following conditions (for all $u, 0,1 \in L^{*}$, where $u=\left(u_{1}, u_{2}\right), \mathbf{0}=0_{L^{*}}=$ $(0,0)$, and $\left.\mathbf{1}=1_{L^{*}}=(1,0)\right)$ :

$\left(\varphi_{1}\right) \varphi(u, \mathbf{1}, u, \mathbf{1}, \mathbf{1}, u)<_{L^{*}} \mathbf{0}$, for all $u>_{L^{*}} \mathbf{0}$;

$\left(\varphi_{2}\right) \varphi(u, \mathbf{1}, \mathbf{1}, u, u, \mathbf{1})<_{L^{*}} \mathbf{0}$, for all $u>_{L^{*}} \mathbf{0}$;

$\left(\varphi_{3}\right) \varphi(u, u, \mathbf{1}, \mathbf{1}, u, u)<_{L^{*}} \mathbf{0}$, for all $u>_{L^{*}} \mathbf{0}$.

Example 23. Define $\varphi\left(t_{1}, t_{2}, t_{3}, t_{4}, t_{5}, t_{6}\right): L^{*^{6}} \rightarrow L^{*}$ as

$$
\varphi\left(t_{1}, t_{2}, t_{3}, t_{4}, t_{5}, t_{6}\right)=t_{1}-\alpha \min \left\{t_{2}, t_{3}, t_{4}, t_{5}, t_{6}\right\},
$$

where $\alpha>1$.

Example 24. Define $\varphi\left(t_{1}, t_{2}, t_{3}, t_{4}, t_{5}, t_{6}\right): L^{*^{6}} \rightarrow L^{*}$ as

$$
\begin{aligned}
\varphi\left(t_{1}, t_{2}, t_{3}, t_{4}, t_{5}, t_{6}\right)= & t_{1}^{2}-\alpha_{1} \min \left\{t_{2}^{2}, t_{3}^{2}, t_{4}^{2}\right\} \\
& -\alpha_{2} \min \left\{t_{3} t_{6}, t_{4} t_{5}\right\},
\end{aligned}
$$

where $\alpha_{1}, \alpha_{2}>0, \alpha_{1}+\alpha_{2}>1$, and $\alpha_{1} \geq 1$.

Example 25. Define $\varphi\left(t_{1}, t_{2}, t_{3}, t_{4}, t_{5}, t_{6}\right): L^{*^{6}} \rightarrow L^{*}$ as

$$
\varphi\left(t_{1}, t_{2}, t_{3}, t_{4}, t_{5}, t_{6}\right)=t_{1}^{3}-\alpha \min \left\{t_{1}^{2} t_{2}, t_{1} t_{3} t_{4}, t_{5}^{2} t_{6}, t_{5} t_{6}^{2}\right\},
$$

where $\alpha>1$.

Example 26. Define $\varphi\left(t_{1}, t_{2}, t_{3}, t_{4}, t_{5}, t_{6}\right): L^{*^{6}} \rightarrow L^{*}$ as

$$
\varphi\left(t_{1}, t_{2}, t_{3}, t_{4}, t_{5}, t_{6}\right)=t_{1}^{3}-\alpha \frac{t_{3}^{2} t_{4}^{2}+t_{5}^{2} t_{6}^{2}}{t_{2}+t_{3}+t_{4}},
$$

where $\alpha \geq 3 / 2$.

Example 27. Define $\varphi\left(t_{1}, t_{2}, t_{3}, t_{4}, t_{5}, t_{6}\right): L^{*^{6}} \rightarrow L^{*}$ as

$$
\begin{aligned}
\varphi\left(t_{1}, t_{2}, t_{3}, t_{4}, t_{5}, t_{6}\right)= & \left(1+\alpha t_{2}\right) t_{1}-\alpha \min \left\{t_{3} t_{4}, t_{5} t_{6}\right\} \\
& -\psi\left(\min \left\{t_{2}, t_{3}, t_{4}, t_{5}, t_{6}\right\}\right),
\end{aligned}
$$

where $\alpha \geq 0$ and $\psi: L^{*} \rightarrow L^{*}$ is a continuous function such that $\psi(s)>_{L^{*}} s$ for all $s \in L^{*} \backslash\{\mathbf{0}, \mathbf{1}\}$. 
Example 28. Define $\varphi\left(t_{1}, t_{2}, t_{3}, t_{4}, t_{5}, t_{6}\right): L^{*^{6}} \rightarrow L^{*}$ as

$$
\varphi\left(t_{1}, t_{2}, t_{3}, t_{4}, t_{5}, t_{6}\right)=t_{1}^{2}-\alpha \frac{t_{2}^{2}+t_{3}^{2}+t_{4}^{2}}{t_{5}+t_{6}}
$$

where $\alpha \geq 2$

Example 29. Define $\varphi\left(t_{1}, t_{2}, t_{3}, t_{4}, t_{5}, t_{6}\right): L^{*^{6}} \rightarrow L^{*}$ as

$$
\varphi\left(t_{1}, t_{2}, t_{3}, t_{4}, t_{5}, t_{6}\right)=t_{1}-\psi\left(\min \left\{t_{2}, t_{3}, t_{4}, t_{5}, t_{6}\right\}\right),
$$

where $\psi: L^{*} \rightarrow L^{*}$ is a continuous function such that $\psi(s)>_{L^{*}} s$ for all $s \in L^{*} \backslash\{\mathbf{0}, \mathbf{1}\}$.

Example 30. Define $\varphi\left(t_{1}, t_{2}, t_{3}, t_{4}, t_{5}, t_{6}\right): L^{*^{6}} \rightarrow L^{*}$ as

$$
\varphi\left(t_{1}, t_{2}, t_{3}, t_{4}, t_{5}, t_{6}\right)=t_{1}^{3}-\alpha \frac{t_{3}^{2} t_{4}^{2}}{t_{2}+t_{5}+t_{6}}
$$

where $\alpha \geq 3$.

Example 31. Define $\varphi\left(t_{1}, t_{2}, t_{3}, t_{4}, t_{5}, t_{6}\right): L^{*^{6}} \rightarrow L^{*}$ as

$$
\varphi\left(t_{1}, t_{2}, t_{3}, t_{4}, t_{5}, t_{6}\right)=t_{1}^{2}-\alpha_{1} \min \left\{t_{2}^{2}, t_{3}^{2}, t_{4}^{2}\right\}-\alpha_{2} \frac{t_{5}}{t_{5}+t_{6}}
$$

where $\alpha_{1} \geq 1$ and $\alpha_{2}>0$.

Example 32. Define $\varphi\left(t_{1}, t_{2}, t_{3}, t_{4}, t_{5}, t_{6}\right): L^{*^{6}} \rightarrow L^{*}$ as

$$
\varphi\left(t_{1}, t_{2}, t_{3}, t_{4}, t_{5}, t_{6}\right)=t_{1}^{2}-\alpha_{1} \min \left\{t_{2}^{2}, t_{5}^{2}, t_{6}^{2}\right\}-\alpha_{2} \frac{t_{3}}{t_{3}+t_{4}},
$$

where $\alpha_{1} \geq 1$ and $\alpha_{2}>0$.

Example 33. Define $\varphi\left(t_{1}, t_{2}, t_{3}, t_{4}, t_{5}, t_{6}\right): L^{*^{6}} \rightarrow L^{*}$ as

$$
\begin{aligned}
\varphi\left(t_{1}, t_{2}, t_{3}, t_{4}, t_{5}, t_{6}\right)= & t_{1}-\alpha_{1} t_{2}-\alpha_{2} t_{3} \\
& -\alpha_{3} t_{4}-\alpha_{4} t_{5}-\alpha_{5} t_{6},
\end{aligned}
$$

where $\alpha_{1}, \alpha_{2}, \alpha_{3}, \alpha_{4}, \alpha_{5}>0, \alpha_{2}+\alpha_{5} \geq 1, \alpha_{3}+\alpha_{4} \geq 1$, and $\alpha_{1}+\alpha_{4}+\alpha_{5} \geq 1$.

On the lines of Tanveer et al. [22], let $\Phi$ be the set of all continuous functions $\phi\left(t_{1}, t_{2}, t_{3}, t_{4}, t_{5}, t_{6}\right): L^{*^{6}} \rightarrow L^{*}$, satisfying (for all $u, v, \mathbf{1} \in L^{*}$, where $u=\left(u_{1}, u_{2}\right), v=\left(v_{1}, v_{2}\right)$, and $\left.\mathbf{1}=1_{L^{*}}=(1,0)\right)$ the following:

$\left(\phi_{1}\right)$ for all $u, v>_{L^{*}} \mathbf{0}, \phi(u, v, u, v, v, u) \geq_{L^{*}} \mathbf{0}$ or $\phi(u, v, v$, $u, u, v) \geq_{L^{*}} \mathbf{0}$ implies that $u \geq_{L^{*}} v$;

$\left(\phi_{2}\right) \phi(u, u, \mathbf{1}, \mathbf{1}, u, u) \geq_{L^{*}} \mathbf{0}$ implies that $u \geq_{L^{*}} \mathbf{1}$.

Example 34. Define $\phi\left(t_{1}, t_{2}, t_{3}, t_{4}, t_{5}, t_{6}\right)=18 t_{1}-16 t_{2}+8 t_{3}-$ $10 t_{4}+t_{5}-t_{6}$. Then $\phi \in \Phi$.

Example 35. Define $\phi\left(t_{1}, t_{2}, t_{3}, t_{4}, t_{5}, t_{6}\right)=t_{1}-(1 / 2) t_{2}-$ $(5 / 6) t_{3}+(1 / 3) t_{4}+t_{5}-t_{6}$. Then $\phi \in \Phi$.
Here, it can be pointed out that the abovementioned classes of functions, namely, $\Psi$ and $\Phi$, are independent to each other as the implicit function $\varphi\left(t_{1}, t_{2}, t_{3}, t_{4}, t_{5}, t_{6}\right)=t_{1}-$ $\alpha \min \left\{t_{2}, t_{3}, t_{4}, t_{5}, t_{6}\right\}$ (where $\alpha>1$ and $\varphi \in \Psi$ ) does not belong to $\Phi$ as $\varphi(u, u, \mathbf{1}, \mathbf{1}, u, u)<_{L^{*}} \mathbf{0}$, for all $u>_{L^{*}} \mathbf{0}$, while the implicit function $\phi\left(t_{1}, t_{2}, t_{3}, t_{4}, t_{5}, t_{6}\right)=15 t_{1}-13 t_{2}+5 t_{3}-$ $7 t_{4}+t_{5}-t_{6}$ (where $\phi \in \Phi$ ) does not belong to $\Psi$ as $\phi(u, v, u, v, v, u)=0$ implies $u=v$ instead of $u>_{L^{*}} v$.

For an extensive collection of implicit relations on different settings, we refer to [41-45].

\section{Results}

Before proving our main results, we observe the following.

Lemma 36. Let $A, B, S$, and $T$ be self-mappings of a modified $\operatorname{IFMS}\left(X, \mathscr{M}_{M, N}, \mathscr{T}\right)$. Suppose that

(1) the pair $(A, S)$ satisfies the $\left(C L R_{S}\right)$ property (or $(B, T)$ satisfies the $\left(C L R_{T}\right)$ property),

(2) $A(X) \subset T(X)($ or $B(X) \subset S(X))$,

(3) $T(X)($ or $S(X))$ is a closed subset of $X$,

(4) $\left\{B y_{n}\right\}$ converges for every sequence $\left\{y_{n}\right\}$ in $X$ whenever $\left\{T y_{n}\right\}$ converges (or $\left\{A x_{n}\right\}$ converges for every sequence $\left\{x_{n}\right\}$ in $X$ whenever $\left\{S x_{n}\right\}$ converges),

(5) for all $x, y \in X$ and $\varphi \in \Psi$

$\varphi\left(\begin{array}{l}\mathscr{M}_{M, N}(A x, B y, t), \mathscr{M}_{M, N}(S x, T y, t), \mathscr{M}_{M, N}(A x, S x, t), \\ \mathscr{M}_{M, N}(B y, T y, t), \mathscr{M}_{M, N}(S x, B y, t), \mathscr{M}_{M, N}(A x, T y, t)\end{array}\right) \geq_{L^{*}} \mathbf{0 .}$.

Then the pairs $(A, S)$ and $(B, T)$ share the $\left(C L R_{S T}\right)$ property.

Proof. If the pair $(A, S)$ enjoys the $\left(\mathrm{CLR}_{S}\right)$ property with respect to mapping $S$, then there exists a sequence $\left\{x_{n}\right\}$ in $X$ such that

$$
\lim _{n \rightarrow \infty} \mathscr{M}_{M, N}\left(A x_{n}, z, t\right)=\lim _{n \rightarrow \infty} \mathscr{M}_{M, N}\left(S x_{n}, z, t\right)=1_{L^{*}},
$$

where $z \in S(X)$. Since $A(X) \subset T(X)$, for each sequence $\left\{x_{n}\right\}$, there exists a sequence $\left\{y_{n}\right\}$ in $X$ such that $A x_{n}=T y_{n}$. Therefore, due to closedness of $T(X)$,

$$
\lim _{n \rightarrow \infty} \mathscr{M}_{M, N}\left(T y_{n}, z, t\right)=\lim _{n \rightarrow \infty} \mathscr{M}_{M, N}\left(A x_{n}, z, t\right)=1_{L^{*}}
$$

where $z \in S(X) \cap T(X)$. Thus, in all, we have $A x_{n} \rightarrow z$, $S x_{n} \rightarrow z$, and $T y_{n} \rightarrow z$ as $n \rightarrow \infty$. Moreover, in view of (4), $\left\{B y_{n}\right\}$ converges. Now, we show that $B y_{n} \rightarrow z$ as $n \rightarrow \infty$. On using inequality (33) with $x=x_{n}, y=y_{n}$, we have

$\varphi\left(\begin{array}{c}\mathscr{M}_{M, N}\left(A x_{n}, B y_{n}, t\right), \mathscr{M}_{M, N}\left(S x_{n}, T y_{n}, t\right), \mathscr{M}_{M, N}\left(A x_{n}, S x_{n}, t\right), \\ \mathscr{M}_{M, N}\left(B y_{n}, T y_{n}, t\right), \mathscr{M}_{M, N}\left(S x_{n}, B y_{n}, t\right), \mathscr{M}_{M, N}\left(A x_{n}, T y_{n}, t\right)\end{array}\right) \geq_{L^{*}} \mathbf{0}$.

Let, on contrary, $B y_{n} \rightarrow l(\neq z)$ as $n \rightarrow \infty$. Then, on making $n \rightarrow \infty$, we get

$$
\varphi\left(\begin{array}{c}
\mathscr{M}_{M, N}(z, l, t), \mathscr{M}_{M, N}(z, z, t), \mathscr{M}_{M, N}(z, z, t), \\
\mathscr{M}_{M, N}(l, z, t), \mathscr{M}_{M, N}(z, l, t), \mathscr{M}_{M, N}(z, z, t)
\end{array}\right) \geq_{L^{*}} \mathbf{0},
$$


or, equivalently,

$$
\varphi\left(\mathscr{M}_{M, N}(z, l, t), \mathbf{1}, \mathbf{1}, \mathscr{M}_{M, N}(l, z, t), \mathscr{M}_{M, N}(z, l, t), \mathbf{1}\right) \geq_{L^{*}} \mathbf{0},
$$

which is a contradiction to $\left(\varphi_{2}\right)$. Hence $\mathscr{M}_{M, N}(z, l, t)=\mathbf{1}$, that is, $z=l$, which shows that $B y_{n} \rightarrow z$ as $n \rightarrow \infty$. Hence both the pairs $(A, S)$ and $(B, T)$ share the $\left(\operatorname{CLR}_{S T}\right)$ property. This concludes the proof.

Remark 37. In general, the converse of Lemma 36 is not true. For a counter example, one can see Example 42.

Now, we state and prove our first main result as follows.

Theorem 38. Let $A, B, S$, and T be self-mappings of a modified IFMS $\left(X, \mathscr{M}_{M, N}, \mathscr{T}\right)$ satisfying inequality (33) of Lemma 36. If the pairs $(A, S)$ and $(B, T)$ share the $\left(C L R_{S T}\right)$ property, then $(A, S)$ and $(B, T)$ have a coincidence point each. Moreover, $A, B$, $S$, and $T$ have a unique common fixed point provided both the pairs $(A, S)$ and $(B, T)$ are weakly compatible.

Proof. Since the pairs $(A, S)$ and $(B, T)$ satisfy the $\left(\operatorname{CLR}_{S T}\right)$ property, there exist two sequences $\left\{x_{n}\right\}$ and $\left\{y_{n}\right\}$ in $X$ such that

$$
\begin{aligned}
\lim _{n \rightarrow \infty} \mathscr{M}_{M, N}\left(A x_{n}, z, t\right) & =\lim _{n \rightarrow \infty} \mathscr{M}_{M, N}\left(S x_{n}, z, t\right) \\
& =\lim _{n \rightarrow \infty} \mathscr{M}_{M, N}\left(B y_{n}, z, t\right) \\
& =\lim _{n \rightarrow \infty} \mathscr{M}_{M, N}\left(T y_{n}, z, t\right)=1_{L^{*}},
\end{aligned}
$$

where $z \in S(X) \cap T(X)$. Since $z \in S(X)$, there exists a point $w \in X$ such that $S w=z$. We show that $A w=S w$. If not, then using inequality (33) with $x=w, y=y_{n}$, we get

$$
\varphi\left(\begin{array}{l}
\mathscr{M}_{M, N}\left(A w, B y_{n}, t\right), \mathscr{M}_{M, N}\left(S w, T y_{n}, t\right), \mathscr{M}_{M, N}(A w, S w, t), \\
\mathscr{M}_{M, N}\left(B y_{n}, T y_{n}, t\right), \mathscr{M}_{M, N}\left(S w, B y_{n}, t\right), \mathscr{M}_{M, N}\left(A w, T y_{n}, t\right)
\end{array}\right) \geq_{L^{*}} \mathbf{0},
$$

which, on making $n \rightarrow \infty$, reduces to

$$
\varphi\left(\begin{array}{c}
\mathscr{M}_{M, N}(A w, z, t), \mathscr{M}_{M, N}(z, z, t), \mathscr{M}_{M, N}(A w, z, t), \\
\mathscr{M}_{M, N}(z, z, t), \mathscr{M}_{M, N}(z, z, t), \mathscr{M}_{M, N}(A w, z, t)
\end{array}\right) \geq_{L^{*}} \mathbf{0}
$$

so that

$$
\varphi\left(\mathscr{M}_{M, N}(A w, z, t), \mathbf{1}, \mathscr{M}_{M, N}(A w, z, t), \mathbf{1}, \mathbf{1}, \mathscr{M}_{M, N}(A w, z, t)\right) \geq_{L^{*}} \mathbf{0}
$$

a contradiction to $\left(\varphi_{1}\right)$. Hence $\mathscr{M}_{M, N}(A w, z, t)=\mathbf{1}$; that is, $A w=S w=z$. Therefore, $w$ is a coincidence point of the pair $(A, S)$.

Also $z \in T(X)$; there exists a point $v \in X$ such that $T v=z$. We assert that $B v=T v$. If not, then using inequality (33) with $x=w, y=v$, we get

$$
\varphi\left(\begin{array}{c}
\mathscr{M}_{M, N}(A w, B v, t), \mathscr{M}_{M, N}(S w, T v, t), \mathscr{M}_{M, N}(A w, S w, t), \\
\mathscr{M}_{M, N}(B v, T v, t), \mathscr{M}_{M, N}(S w, B v, t), \mathscr{M}_{M, N}(A w, T v, t)
\end{array}\right) \geq_{L^{*}} \mathbf{0}
$$

so that

$$
\varphi\left(\begin{array}{l}
\mathscr{M}_{M, N}(z, B v, t), \mathscr{M}_{M, N}(z, z, t), \mathscr{M}_{M, N}(z, z, t), \\
\mathscr{M}_{M, N}(B v, z, t), \mathscr{M}_{M, N}(z, B v, t), \mathscr{M}_{M, N}(z, z, t)
\end{array}\right) z_{L^{*}} \mathbf{0}
$$

or

$$
\varphi\left(\mathscr{M}_{M, N}(z, B v, t), \mathbf{1}, \mathbf{1}, \mathscr{M}_{M, N}(B v, z, t), \mathscr{M}_{M, N}(z, B v, t), \mathbf{1}\right) \geq_{L^{*}} \mathbf{0},
$$

a contradiction to $\left(\varphi_{2}\right)$. Hence $\mathscr{M}_{M, N}(z, B v, t)=\mathbf{1}$, and so $B v=T v=z$, which shows that $v$ is a coincidence point of the pair $(B, T)$.

Since the pair $(A, S)$ is weakly compatible and $A w=S w$, hence $A z=A S w=S A w=S z$. Now, we show that $z$ is a common fixed point of the pair $(A, S)$. Suppose that $A z \neq z$; using inequality (33) with $x=z, y=v$, we have

$$
\varphi\left(\begin{array}{c}
\mathscr{M}_{M, N}(A z, B v, t), \mathscr{M}_{M, N}(S z, T v, t), \mathscr{M}_{M, N}(A z, S z, t), \\
\mathscr{M}_{M, N}(B v, T v, t), \mathscr{M}_{M, N}(S z, B v, t), \mathscr{M}_{M, N}(A z, T v, t)
\end{array}\right) \geq_{L^{*}} \mathbf{0}
$$

so that

$$
\varphi\left(\begin{array}{c}
\mathscr{M}_{M, N}(A z, z, t), \mathscr{M}_{M, N}(A z, z, t), \mathscr{M}_{M, N}(A z, A z, t), \\
\mathscr{M}_{M, N}(z, z, t), \mathscr{M}_{M, N}(A z, z, t), \mathscr{M}_{M, N}(A z, z, t)
\end{array}\right) \geq_{L^{*}} \mathbf{0}
$$

or

$$
\varphi\left(\begin{array}{c}
\mathscr{M}_{M, N}(A z, z, t), \mathscr{M}_{M, N}(A z, z, t), \mathbf{1}, \mathbf{1}, \\
\mathscr{M}_{M, N}(A z, z, t), \mathscr{M}_{M, N}(A z, z, t)
\end{array}\right) \geq_{L^{*}} \mathbf{0}
$$

a contradiction to $\left(\varphi_{3}\right)$ yielding thereby $A z=z=S z$ which shows that $z$ is a common fixed point of the pair $(A, S)$.

Also the pair $(B, T)$ is weakly compatible, and $B v=T v$; therefore, $B z=B T v=T B v=T z$. Suppose that $B z \neq z$; then using inequality (33) with $x=w, y=z$, we have

$$
\varphi\left(\begin{array}{c}
\mathscr{M}_{M, N}(A w, B z, t), \mathscr{M}_{M, N}(S w, T z, t), \mathscr{M}_{M, N}(A w, S w, t), \\
\mathscr{M}_{M, N}(B z, T z, t), \mathscr{M}_{M, N}(S w, B z, t), \mathscr{M}_{M, N}(A w, T z, t)
\end{array}\right) \geq_{L^{*}} \mathbf{0},
$$

so that

$$
\varphi\left(\begin{array}{c}
\mathscr{M}_{M, N}(z, B z, t), \mathscr{M}_{M, N}(z, B z, t), \mathscr{M}_{M, N}(z, z, t), \\
\mathscr{M}_{M, N}(B z, B z, t), \mathscr{M}_{M, N}(z, B z, t), \mathscr{M}_{M, N}(z, B z, t)
\end{array}\right) \geq_{L^{*}} \mathbf{0}
$$

or

$$
\varphi\left(\begin{array}{c}
\mathscr{M}_{M, N}(z, B z, t), \mathscr{M}_{M, N}(z, B z, t), \mathbf{1}, \mathbf{1}, \\
\mathscr{M}_{M, N}(z, B z, t), \mathscr{M}_{M, N}(z, B z, t)
\end{array}\right) \geq_{L^{*}} \mathbf{0},
$$

a contradiction to $\left(\varphi_{3}\right)$. Therefore, $B z=z=T z$ which shows that $z$ is a common fixed point of the pair $(B, T)$. Hence $z$ is a common fixed point of both the pairs $(A, S)$ and $(B, T)$. Uniqueness of common fixed point is an easy consequence of inequality (33) (owing to condition $\left(\varphi_{3}\right)$ ). This completes the proof.

Remark 39. Theorem 38 improves the corresponding results contained in Imdad et al. [23] as closedness of the underlying subspaces is not required.

Now, we present an example which demonstrates the validity of the hypotheses and degree of generality of our main result over comparable ones from the existing literature. 
Example 40. Let $\left(X, \mathscr{M}_{M, N}, \mathscr{T}\right)$ be a modified IFMS, wherein $X=[5,21), \mathscr{T}(a, b)=\left(a_{1} b_{1}, \min \left\{a_{2}+b_{2}, 1\right\}\right)$ for all $a=\left(a_{1}, a_{2}\right)$ and $b=\left(b_{1}, b_{2}\right) \in L^{*}$ with $\mathscr{M}_{M, N}(x, y, t)=(t /(t+|x-y|), \mid x-$ $y \mid /(t+|x-y|))$ for all $x, y \in X$ and $t>0$. Define four self-mappings $A, B, S$, and $T$ by

$$
\begin{aligned}
& A(x)= \begin{cases}5, & \text { if } x \in\{5\} \cup(9,21) ; \\
20, & \text { if } x \in(5,9],\end{cases} \\
& B(x)= \begin{cases}5, & \text { if } x \in\{5\} \cup(9,21) \\
13, & \text { if } x \in(5,9]\end{cases} \\
& S(x)= \begin{cases}5, & \text { if } x=5 ; \\
10, & \text { if } x \in(5,9] \\
\frac{x+1}{2}, & \text { if } x \in(9,21)\end{cases} \\
& T(x)= \begin{cases}5, & \text { if } x=5 \\
18, & \text { if } x \in(5,9] \\
x-4, & \text { if } x \in(9,21)\end{cases}
\end{aligned}
$$

Define an implicit function $\varphi\left(t_{1}, t_{2}, t_{3}, t_{4}, t_{5}, t_{6}\right): L^{*^{6}} \rightarrow L^{*}$ by

$$
\begin{aligned}
& \varphi\left(t_{1}, t_{2}, t_{3}, t_{4}, t_{5}, t_{6}\right) \\
& =t_{1}-\psi\left(\min \left\{t_{2}, t_{3}, t_{4}, t_{5}, t_{6}\right\}\right),
\end{aligned}
$$

where $\psi: L^{*} \rightarrow L^{*}$ is a continuous function such that $\psi(s)>_{L^{*}} S$ (that is, $\psi(s)=\sqrt{s}$ ) for all $s \in L^{*} \backslash\{\mathbf{0}, \mathbf{1}\}$ and $\varphi \in \Psi$. Hence (53) implies

$$
\begin{aligned}
& \mathscr{M}_{M, N}(A x, B y, t) \\
& \geq_{L^{*}} \sqrt{\min \left\{\begin{array}{c}
\mathscr{M}_{M, N}(S x, T y, t), \mathscr{M}_{M, N}(A x, S x, t), \mathscr{M}_{M, N}(B y, T y, t), \\
\mathscr{M}_{M, N}(S x, B y, t), \mathscr{M}_{M, N}(A x, T y, t)
\end{array}\right\}},
\end{aligned}
$$

for all $x, y \in X$ and $t>0$. With two sequences $\left\{x_{n}\right\}=$ $\{9+1 / n\}_{n \in \mathbb{N}}$ and $\left\{y_{n}\right\}=\{5\}$ (or $\left\{x_{n}\right\}=\{5\},\left\{y_{n}\right\}=$ $\left.\{9+1 / n\}_{n \in \mathbb{N}}\right)$, the pairs $(A, S)$ and $(B, T)$ satisfy the $\left(\operatorname{CLR}_{S T}\right)$ property:

$$
\begin{aligned}
\lim _{n \rightarrow \infty} \mathscr{M}_{M, N}\left(A x_{n}, 5, t\right) & =\lim _{n \rightarrow \infty} \mathscr{M}_{M, N}\left(S x_{n}, 5, t\right) \\
& =\lim _{n \rightarrow \infty} \mathscr{M}_{M, N}\left(B y_{n}, 5, t\right) \\
& =\lim _{n \rightarrow \infty} \mathscr{M}_{M, N}\left(T y_{n}, 5, t\right)=1_{L^{*}},
\end{aligned}
$$

where $5 \in S(X) \cap T(X)$. Also, $A(X)=\{5,20\} \nsubseteq[5,17) \cup\{18\}=$ $T(X)$ and $B(X)=\{5,13\} \nsubseteq[5,11)=S(X)$. By a routine calculation, one can easily verify the inequality (54) for all $x, y \in X$. Thus all the conditions of Theorem 38 are satisfied, and 5 is a unique common fixed point of the pairs $(A, S)$ and $(B, T)$, which also remains a point of coincidence as well. Here, one may notice that all the involved mappings are discontinuous even at their unique common fixed point 5 .

Notice that the subspaces $S(X)$ and $T(X)$ are not closed subspaces of $X$; therefore, the main result contained in Imdad et al. [23] can not be used in the context of this example which establishes the genuineness of our extension.
In the proof of our next theorem, Lemma 36 is utilized.

Theorem 41. Let $A, B, S$, and $T$ be self-mappings of a modified $\operatorname{IFMS}\left(X, \mathscr{M}_{M, N}, \mathscr{T}\right)$ satisfying all the hypotheses of Lemma 36. Then $A, B, S$, and $T$ have a unique common fixed point provided both the pairs $(A, S)$ and $(B, T)$ are weakly compatible.

Proof. In view of Lemma 36, the pairs $(A, S)$ and $(B, T)$ share the $\left(\mathrm{CLR}_{S T}\right)$ property so that there exist two sequences $\left\{x_{n}\right\}$ and $\left\{y_{n}\right\}$ in $X$ such that

$$
\begin{aligned}
\lim _{n \rightarrow \infty} \mathscr{M}_{M, N}\left(A x_{n}, z, t\right) & =\lim _{n \rightarrow \infty} \mathscr{M}_{M, N}\left(S x_{n}, z, t\right) \\
& =\lim _{n \rightarrow \infty} \mathscr{M}_{M, N}\left(B y_{n}, z, t\right) \\
& =\lim _{n \rightarrow \infty} \mathscr{M}_{M, N}\left(T y_{n}, z, t\right)=1_{L^{*}},
\end{aligned}
$$

where $z \in S(X) \cap T(X)$. The rest of the proof can be completed on the lines of the proof of Theorem 38. This completes the proof.

The following example demonstrates the utility of Theorem 41 over Theorem 38.

Example 42. In the setting of Example 40, replace the selfmappings $A, B, S$, and $T$ by the following, besides retaining the rest:

$$
\begin{aligned}
& A(x)= \begin{cases}5, & \text { if } x \in\{5\} \cup(9,21) \\
15, & \text { if } x \in(5,9]\end{cases} \\
& B(x)= \begin{cases}5, & \text { if } x \in\{5\} \cup(9,21) \\
10, & \text { if } x \in(5,9]\end{cases} \\
& S(x)= \begin{cases}5, & \text { if } x=5 \\
11, & \text { if } x \in(5,9] \\
\frac{x+1}{2}, & \text { if } x \in(9,21)\end{cases} \\
& T(x)= \begin{cases}5, & \text { if } x=5 \\
17, & \text { if } x \in(5,9] \\
x-4, & \text { if } x \in(9,21)\end{cases}
\end{aligned}
$$

Then, like the earlier example, it is easy to see that both the pairs $(A, S)$ and $(B, T)$ satisfy the $\left(C L R_{S T}\right)$ property. Consider an implicit function described by Example 40. Also, $A(X)=$ $\{5,15\} \subset[5,17]=T(X)$ and $B(X)=\{5,10\} \subset[5,11]=S(X)$. The pairs $(A, S)$ and $(B, T)$ commute at 5 which is also their common coincidence point. Thus all the conditions of Theorems 41 are satisfied, and 5 is a unique common fixed point of the involved mappings $A, B, S$, and $T$.

Here, it can be pointed out that Theorem 38 is not applicable to this example as both $S(X), T(X)$ are closed subsets of $X$ which demonstrates the situational utility of Theorem 41 over Theorem 38 . 
In view of the earlier demonstrative examples, one can outline the following corollary.

Corollary 43. The conclusions of Lemma 36, Theorem 38, and Theorem 41 remain true if inequality (33) is replaced by one of the following contraction conditions. For all $x, y \in X$ and $t>0$,

$\mathscr{M}_{M, N}(A x, B y, t)$

$\geq_{L^{*}} \alpha \min \left\{\begin{array}{c}\mathscr{M}_{M, N}(S x, T y, t), \mathscr{M}_{M, N}(A x, S x, t), \mathscr{M}_{M, N}(B y, T y, t), \\ \mathscr{M}_{M, N}(S x, B y, t), \mathscr{M}_{M, N}(A x, T y, t)\end{array}\right\}$, where $\alpha>1$,

$$
\begin{aligned}
& \mathscr{M}_{M, N}^{2}(A x, B y, t) \\
& \geq_{L^{*}} \alpha_{1} \min \left\{\mathscr{M}_{M, N}^{2}(S x, T y, t), \mathscr{M}_{M, N}^{2}(A x, S x, t), \mathscr{M}_{M, N}^{2}(B y, T y, t)\right\} \\
& \quad+\alpha_{2} \min \left\{\begin{array}{c}
\mathscr{M}_{M, N}(A x, S x, t) \mathscr{M}_{M, N}(A x, T y, t), \\
\mathscr{M}_{M, N}(B y, T y, t) \mathscr{M}_{M, N}(S x, B y, t)
\end{array}\right\},
\end{aligned}
$$

where $\alpha_{1}, \alpha_{2}>0, \alpha_{1}+\alpha_{2}>1$ and $\alpha_{1} \geq 1$,

$$
\begin{aligned}
& \mathscr{M}_{M, N}^{3}(A x, B y, t) \\
& \geq_{L^{*}} \alpha \min \left\{\begin{array}{c}
\mathscr{M}_{M, N}^{2}(A x, B y, t) \mathscr{M}_{M, N}(S x, T y, t), \\
\mathscr{M}_{M, N}(A x, B y, t) \mathscr{M}_{M, N}(A x, S x, t) \mathscr{M}_{M, N}(B y, T y, t), \\
\mathscr{M}_{M, N}^{2}(S x, B y, t) \mathscr{M}_{M, N}(A x, T y, t), \mathscr{M}_{M, N}(S x, B y, t) \mathscr{M}_{M, N}^{2}(A x, T y, t)
\end{array}\right\},
\end{aligned}
$$

where $\alpha>1$,

$$
\mathscr{M}_{M, N}^{3}(A x, B y, t) \geq_{L^{*}} \alpha \frac{\mathscr{M}_{M, N}^{2}(A x, S x, t) \mathscr{M}_{M, N}^{2}(B y, T y, t)+\mathscr{M}_{M, N}^{2}(S x, B y, t), \mathscr{M}_{M, N}^{2}(A x, T y, t)}{\mathscr{M}_{M, N}(S x, T y, t)+\mathscr{M}_{M, N}(A x, S x, t)+\mathscr{M}_{M, N}(B y, T y, t)},
$$

where $\alpha \geq 3 / 2$,

$$
\begin{aligned}
& \left(1+\alpha \mathscr{M}_{M, N}(S x, T y, t)\right) \mathscr{M}_{M, N}(A x, B y, t) \\
& \geq_{L^{*}} \alpha \min \left\{\begin{array}{c}
\mathscr{M}_{M, N}(A x, S x, t) \mathscr{M}_{M, N}(B y, T y, t), \\
\mathscr{M}_{M, N}(S x, B y, t) \mathscr{M}_{M, N}(A x, T y, t)
\end{array}\right\} \\
& -\psi\left(\min \left\{\begin{array}{c}
\mathscr{M}_{M, N}(S x, T y, t), \mathscr{M}_{M, N}(A x, S x, t), \\
\mathscr{M}_{M, N}(B y, T y, t), \mathscr{M}_{M, N}(S x, B y, t), \\
\mathscr{M}_{M, N}(A x, T y, t)
\end{array}\right\}\right),
\end{aligned}
$$

where $\alpha \geq 0$ and $\psi: L^{*} \rightarrow L^{*}$ is a continuous function such that $\psi(s)>_{L^{*}} s$ for all $s \in L^{*} \backslash\{\mathbf{0}, \mathbf{1}\}$,

$$
\begin{aligned}
& \mathscr{M}_{M, N}^{2}(A x, B y, t) \\
& \geq_{L^{*}} \alpha \frac{\mathscr{M}_{M, N}^{2}(S x, T y, t)+\mathscr{M}_{M, N}^{2}(A x, S x, t)+\mathscr{M}_{M, N}^{2}(B y, T y, t)}{\mathscr{M}_{M, N}(S x, B y, t)+\mathscr{M}_{M, N}(A x, T y, t)},
\end{aligned}
$$

where $\alpha \geq 2$,

$\mathscr{M}_{M, N}(A x, B y, t)$

$z_{L^{*}} \psi\left(\min \left\{\begin{array}{c}\mathscr{M}_{M, N}(S x, T y, t), \mathscr{M}_{M, N}(A x, S x, t), \mathscr{M}_{M, N}(B y, T y, t), \\ \mathscr{M}_{M, N}(S x, B y, t), \mathscr{M}_{M, N}(A x, T y, t)\end{array}\right\}\right)$

where $\psi: L^{*} \rightarrow L^{*}$ is a continuous function such that $\psi(s)>_{L^{*}} s$ for all $s \in L^{*} \backslash\{\mathbf{0}, \mathbf{1}\}$,

$$
\begin{aligned}
& \mathscr{M}_{M, N}^{3}(A x, B y, t) \\
& \geq_{L^{*}} \alpha \frac{\mathscr{M}_{M, N}^{2}(A x, S x, t) \mathscr{M}_{M, N}^{2}(B y, T y, t)}{\mathscr{M}_{M, N}(S x, T y, t)+\mathscr{M}_{M, N}(S x, B y, t)+\mathscr{M}_{M, N}(A x, T y, t)},
\end{aligned}
$$

where $\alpha \geq 3$,

$$
\begin{aligned}
& \mathscr{M}_{M, N}^{2}(A x, B y, t) \\
& \geq_{L^{*}} \alpha_{1} \min \left\{\mathscr{M}_{M, N}^{2}(S x, T y, t), \mathscr{M}_{M, N}^{2}(A x, S x, t), \mathscr{M}_{M, N}^{2}(B y, T y, t)\right\} \\
& \quad+\alpha_{2} \frac{\mathscr{M}_{M, N}(S x, B y, t)}{\mathscr{M}_{M, N}(S x, B y, t)+\mathscr{M}_{M, N}(A x, T y, t)},
\end{aligned}
$$

where $\alpha_{1} \geq 1$ and $\alpha_{2}>0$,

$$
\begin{aligned}
& \mathscr{M}_{M, N}^{2}(A x, B y, t) \\
& \geq_{L^{*}} \alpha_{1} \min \left\{\mathscr{M}_{M, N}^{2}(S x, T y, t), \mathscr{M}_{M, N}^{2}(S x, B y, t), \mathscr{M}_{M, N}^{2}(A x, T y, t)\right\} \\
& \quad+\alpha_{2} \frac{\mathscr{M}_{M, N}(A x, S x, t)}{\mathscr{M}_{M, N}(A x, S x, t)+\mathscr{M}_{M, N}(B y, T y, t)},
\end{aligned}
$$

where $\alpha_{1} \geq 1$ and $\alpha_{2}>0$,

$$
\begin{aligned}
& \mathscr{M}_{M, N}(A x, B y, t) \\
& \geq_{L^{*}} \alpha_{1} \mathscr{M}_{M, N}(S x, T y, t)+\alpha_{2} \mathscr{M}_{M, N}(A x, S x, t)+\alpha_{3} \mathscr{M}_{M, N}(B y, T y, t) \\
& \quad+\alpha_{4} \mathscr{M}_{M, N}(S x, B y, t)+\alpha_{5} \mathscr{M}_{M, N}(A x, T y, t),
\end{aligned}
$$

where $\alpha_{1}, \alpha_{2}, \alpha_{3}, \alpha_{4}, \alpha_{5}>0, \alpha_{2}+\alpha_{5} \geq 1, \alpha_{3}+\alpha_{4} \geq 1$, and $\alpha_{1}+\alpha_{4}+\alpha_{5} \geq 1$

Proof. The proof for each inequality (58)-(68) easily follows from Theorem 38 in view of Examples 23-33. 
Remark 44. Corollary 43 improves and generalizes a multitude of well-known results especially those contained in $[8$, $10,11,17,19,22,23]$.

Now we state the next theorem for another independent class of implicit functions $\phi \in \Phi$ utilized in Tanveer et al. [22].

Theorem 45. Let $A, B, S$, and $T$ be self-mappings of a modified $\operatorname{IFMS}\left(X, \mathscr{M}_{M, N}, \mathscr{T}\right)$ satisfying

$$
\phi\left(\begin{array}{c}
\mathscr{M}_{M, N}(A x, B y, t), \mathscr{M}_{M, N}(S x, T y, t), \mathscr{M}_{M, N}(A x, S x, t), \\
\mathscr{M}_{M, N}(B y, T y, t), \mathscr{M}_{M, N}(S x, B y, t), \mathscr{M}_{M, N}(A x, T y, t)
\end{array}\right) z_{L^{*}} \mathbf{0},
$$

for all $x, y \in X$ and $\phi \in \Phi$. If the pairs $(A, S)$ and $(B, T)$ share the $\left(C L R_{S T}\right)$ property, then $(A, S)$ and $(B, T)$ have a coincidence point each. Moreover, $A, B, S$, and $T$ have a unique common fixed point provided both the pairs $(A, S)$ and $(B, T)$ are weakly compatible.

Proof. The proof of this theorem can be completed on the lines of the proof of Theorem 38; hence we skip the details.

Example 46. In the setting of Example 40, one can define an implicit function $\phi\left(t_{1}, t_{2}, t_{3}, t_{4}, t_{5}, t_{6}\right): L^{*^{6}} \rightarrow L^{*}$ by $\phi\left(t_{1}, t_{2}, t_{3}, t_{4}, t_{5}, t_{6}\right)=18 t_{1}-16 t_{2}+8 t_{3}-10 t_{4}+t_{5}-t_{6}$, where $\phi \in \Phi$ (besides retaining the rest). Hence the pairs $(A, S)$ and $(B, T)$ enjoy the $\left(\mathrm{CLR}_{S T}\right)$ property. Thus all the conditions of Theorem 45 are satisfied, and 5 is a unique common fixed point of the pairs $(A, S)$ and $(B, T)$.

By choosing $A, B, S$, and $T$ suitably, we can deduce corollaries involving two as well as three self-mappings. For the sake naturality, we only derive the following corollary involving a pair of self-mappings.

Corollary 47. Let $A$ and $S$ be self-mappings of a modified $\operatorname{IFMS}\left(X, \mathscr{M}_{M, N}, \mathscr{T}\right)$. Suppose that

(1) the pair $(A, S)$ satisfies the $\left(C L R_{S}\right)$ property,

(2) for all $x, y \in X$ and $\varphi \in \Psi$,

$$
\varphi\left(\begin{array}{c}
\mathscr{M}_{M, N}(A x, A y, t), \mathscr{M}_{M, N}(S x, S y, t), \mathscr{M}_{M, N}(A x, S x, t), \\
\mathscr{M}_{M, N}(A y, S y, t), \mathscr{M}_{M, N}(S x, A y, t), \mathscr{M}_{M, N}(A x, S y, t)
\end{array}\right) \geq_{L^{*}} \mathbf{0} .
$$

Then $(A, S)$ has a coincidence point. Moreover, if the pair $(A, S)$ is weakly compatible then the pair has a unique common fixed point in $X$.

As an application of Theorem 38, we have the following result involving four finite families of self-mappings.

Theorem 48. Let $\left\{A_{i}\right\}_{i=1}^{m},\left\{B_{j}\right\}_{r=1}^{n},\left\{S_{k}\right\}_{k=1}^{p}$, and $\left\{T_{l}\right\}_{l=1}^{q}$ be four finite families of self-mappings of a modified IFMS (X, $\mathscr{M}_{M, N}$, T) with $A=A_{1} A_{2} \cdots A_{m}, B=B_{1} B_{2} \cdots B_{n}, S=S_{1} S_{2} \cdots S_{p}$, and $T=T_{1} T_{2} \cdots T_{q}$ satisfying the condition (33). Suppose that the pairs $(A, S)$ and $(B, T)$ enjoy the $\left(C L R_{S T}\right)$ property; then $(A, S)$ and $(B, T)$ have a point of coincidence each.
Moreover $\left\{A_{i}\right\}_{i=1}^{m},\left\{B_{j}\right\}_{j=1}^{n},\left\{S_{k}\right\}_{k=1}^{p}$, and $\left\{T_{l}\right\}_{l=1}^{q}$ have a unique common fixed point if the families $\left(\left\{A_{i}\right\},\left\{S_{k}\right\}\right)$ and $\left(\left\{B_{r}\right\},\left\{T_{h}\right\}\right)$ commute pairwise, wherein $i \in\{1,2, \ldots, m\}, k \in$ $\{1,2, \ldots, p\}, j \in\{1,2, \ldots, n\}$, and $l \in\{1,2, \ldots, q\}$.

Proof. The proof of this theorem can be completed on the lines of the proof of a similar theorem contained in Imdad et al. [40].

Remark 49. A result similar to Theorem 48 can be outlined in respect of Theorem 38. Notice that Theorem 48 generalizes certain results of Sharma and Deshpande [13].

Now, we indicate that Theorem 48 can be utilized to derive common fixed point theorems for any finite number of mappings. As a sample, we derive a theorem involving five mappings by setting one family of two members while the remaining three of single members.

Corollary 50. Let $A, B, R, S$, and $T$ be self-mappings of a modified IFMS $\left(X, \mathscr{M}_{M, N}, \mathscr{T}\right)$. Suppose that

(1) the pairs $(A, S R)$ and $(B, T)$ share the $\left(C L R_{(S R)(T)}\right)$ property,

(2) for all $x, y \in X$ and $\varphi \in \Psi$,

$\varphi\left(\begin{array}{c}\mathscr{M}_{M, N}(A x, B y, t), \mathscr{M}_{M, N}(S R x, T y, t), \mathscr{M}_{M, N}(A x, S R x, t), \\ \mathscr{M}_{M, N}(B y, T y, t), \mathscr{M}_{M, N}(S R x, B y, t), \mathscr{M}_{M, N}(A x, T y, t)\end{array}\right) z_{L^{*}} \mathbf{0}$.

Then $(A, S R)$ and $(B, T)$ have a coincidence point each. Moreover, $A, B, R, S$, and $T$ have a unique common fixed point provided both the pairs $(A, S R)$ and $(B, T)$ commute pairwise; that is, $A S=S A, A R=R A, S R=R S$, and $B T=T B$.

Similarly, we can derive a common fixed point theorem for six mappings by setting two families of two members while the rest two of single members.

Corollary 51. Let $A, B, H, R, S$, and $T$ be self-mappings of a modified IFMS $\left(X, \mathscr{M}_{M, N}, \mathscr{T}\right)$. Suppose that

(1) the pairs $(A, S R)$ and $(B, T H)$ share the $\left(C L R_{(S R)(T H)}\right)$ property,

(2) for all $x, y \in X$ and $\varphi \in \Psi$,

$\varphi\left(\begin{array}{c}\mathscr{M}_{M, N}(A x, B y, t), \mathscr{M}_{M, N}(S R x, T H y, t), \mathscr{M}_{M, N}(A x, S R x, t), \\ \mathscr{M}_{M, N}(B y, T H y, t), \mathscr{M}_{M, N}(S R x, B y, t), \mathscr{M}_{M, N}(A x, T H y, t)\end{array}\right) \geq_{L^{*}} \mathbf{0}$.

Then $(A, S R)$ and $(B, T H)$ have a coincidence point each. Moreover, $A, B, H, R, S$, and $T$ have a unique common fixed point provided both the pairs $(A, S R)$ and $(B, T H)$ commute pairwise; that is, $A S=S A, A R=R A, S R=R S, B T=T B$, $B H=H B$, and $T H=H T$.

By setting $A_{1}=A_{2}=\cdots=A_{m}=A, B_{1}=B_{2}=\cdots=$ $B_{n}=B, S_{1}=S_{2}=\cdots=S_{p}=S$, and $T_{1}=T_{2}=\cdots=T_{q}=T$ in Theorem 48 , we deduce the following. 
Corollary 52. Let $A, B, S$, and $T$ be self-mappings of a modified IFMS $\left(X, \mathscr{M}_{M, N}, \mathscr{T}\right)$. Suppose that

(1) the pairs $\left(A^{m}, S^{p}\right)$ and $\left(B^{n}, T^{q}\right)$ share the $\left(C L R_{S^{p} T^{q}}\right)$ property,

(2) for all $x, y \in X$ and $\varphi \in \Psi$,

$\varphi\left(\begin{array}{c}\mathscr{M}_{M, N}\left(A^{m} x, B^{n} y, t\right), \mathscr{M}_{M, N}\left(S^{p} x, T^{q} y, t\right), \mathscr{M}_{M, N}\left(A^{m} x, S^{p} x, t\right), \\ \mathscr{M}_{M, N}\left(B^{n} y, T^{q} y, t\right), \mathscr{M}_{M, N}\left(S^{p} x, B^{n} y, t\right), \mathscr{M}_{M, N}\left(A^{m} x, T^{q} y, t\right)\end{array}\right) \geq_{L^{*}} \mathbf{0}$,

where $m, n, p$, and q are fixed positive integers.

Then $A, B, S$, and $T$ have a unique common fixed point provided $A S=S A$ and $B T=T B$.

Remark 53. Corollary 52 is a slight but partial generalization of Theorem 38 as the commutativity requirements (that is, $A S=S A$ and $B T=T B$ ) in this corollary are relatively stronger as compared to weak compatibility in Theorem 38 .

Remark 54. Results similar to Corollary 52 can be derived from Theorem 38 and Corollary 43.

Remark 55. It is noticed that Lemma 36, Theorems 38-48, and Corollaries 43-52 can be also proved for the implicit function $\Phi$, but due to paucity of the space we have opted not to include the entire details.

\section{Conflict of Interests}

The authors declare that they have no conflict of interests.

\section{Acknowledgments}

The authors are thankful to all anonymous referees for their valuable suggestions and critical remarks towards the improvement of this paper. The authors are also very grateful to Professor Erdal Karapnar (Guest Editor of Abstract and Applied Analysis) for his encouragements, especially in the course of two revisions.

\section{References}

[1] L. A. Zadeh, "Fuzzy sets," Information and Computation, vol. 8, pp. 338-353, 1965.

[2] K. T. Atanassov, "Intuitionistic fuzzy sets," Fuzzy Sets and Systems, vol. 20, no. 1, pp. 87-96, 1986.

[3] D. Çoker, "An introduction to intuitionistic fuzzy topological spaces," Fuzzy Sets and Systems, vol. 88, no. 1, pp. 81-89, 1997.

[4] T. K. Mondal and S. K. Samanta, "On intuitionistic gradation of openness," Fuzzy Sets and Systems, vol. 131, no. 3, pp. 323-336, 2002.

[5] J. H. Park, "Intuitionistic fuzzy metric spaces," Chaos, Solitons and Fractals, vol. 22, no. 5, pp. 1039-1046, 2004.

[6] A. George and P. Veeramani, "On some results in fuzzy metric spaces," Fuzzy Sets and Systems, vol. 64, no. 3, pp. 395-399, 1994.

[7] H. M. Abu-Donia and A. A. Nasef, "Common fixed point theorems in intuitionistic fuzzy metric spaces," Mohu Xitong yu Shuxue, vol. 22, no. 2, pp. 100-106, 2008.
[8] C. Alaca, D. Turkoglu, and C. Yildiz, "Fixed points in intuitionistic fuzzy metric spaces," Chaos, Solitons \& Fractals, vol. 29, no. 5, pp. 1073-1078, 2006.

[9] L. B. Ćirić, S. N. Ješić, and J. S. Ume, "The existence theorems for fixed and periodic points of nonexpansive mappings in intuitionistic fuzzy metric spaces," Chaos, Solitons and Fractals, vol. 37, no. 3, pp. 781-791, 2008.

[10] X. Huang, C. Zhu, and X. Wen, "Common fixed point theorems for families of compatible mappings in intuitionistic fuzzy metric spaces," Annali dell'Universitá di Ferrara, vol. 56, no. 2, pp. 305-326, 2010.

[11] B. D. Pant, S. Kumar, and S. Chauhan, "Common fixed point of weakly compatible maps on intuitionistic fuzzy metric spaces," Journal of Advanced Studies in Topology, vol. 1, no. 1, pp. 41-49, 2010.

[12] S. Sedghi, N. Shobe, and A. Aliouche, "Common fixed point theorems in intuitionistic fuzzy metric spaces through conditions of integral type," Applied Mathematics \& Information Sciences, vol. 2, no. 1, pp. 61-82, 2008.

[13] S. Sharma and B. Deshpande, "Common fixed point theorems for finite number of mappings without continuity and compatibility on intuitionistic fuzzy metric spaces," Chaos, Solitons and Fractals, vol. 40, no. 5, pp. 2242-2256, 2009.

[14] W. Sintunavarat, S. Chauhan, and P. Kumam, "Some fixed point results in modofied intuitionistic fuzzy metric spaces," Afrika Matematika, 2013.

[15] W. Sintunavarat and P. Kumam, "Fixed point theorems for a generalized intuitionistic fuzzy contraction in intuitionistic fuzzy metric spaces," Thai Journal of Mathematics, vol. 10, no. 1, pp. 123-135, 2012.

[16] V. Gregori, S. Romaguera, and P. Veeramani, "A note on intuitionistic fuzzy metric spaces," Chaos, Solitons \& Fractals, vol. 28, no. 4, pp. 902-905, 2006.

[17] R. Saadati and J. H. Park, "On the intuitionistic fuzzy topological spaces," Chaos, Solitons and Fractals, vol. 27, no. 2, pp. 331-344, 2006.

[18] G. Jungck, "Compatible mappings and common fixed points," International Journal of Mathematics and Mathematical Sciences, vol. 9, no. 4, pp. 771-779, 1986.

[19] S. Jain, S. Jain, and L. Bahadur Jain, "Compatibility of type (P) in modified intuitionistic fuzzy metric space," Journal of Nonlinear Science and its Applications, vol. 3, no. 2, pp. 96-109, 2010.

[20] G. Jungck and B. E. Rhoades, "Fixed points for set valued functions without continuity," Indian Journal of Pure and Applied Mathematics, vol. 29, no. 3, pp. 227-238, 1998.

[21] R. P. Pant, "Common fixed point theorems for contractive maps," Journal of Mathematical Analysis and Applications, vol. 226, no. 1, pp. 251-258, 1998.

[22] M. Tanveer, M. Imdad, D. Gopal, and D. K. Patel, "Common fixed point theorems in modified intuitionistic fuzzy metric spaces with common property (E.A.)," Fixed Point Theory and Applications, vol. 2012, article 36, 2012.

[23] M. Imdad, J. Ali, and M. Hasan, "Common fixed point theorems in modified intuitionistic fuzzy metric spaces," Iranian Journal of Fuzzy Systems, vol. 9, no. 5, pp. 77-92, 2012.

[24] M. Aamri and D. El Moutawakil, "Some new common fixed point theorems under strict contractive conditions," Journal of Mathematical Analysis and Applications, vol. 270, no. 1, pp. 181188, 2002.

[25] Y. Liu, J. Wu, and Z. Li, "Common fixed points of single-valued and multivalued maps," International Journal of Mathematics and Mathematical Sciences, no. 19, pp. 3045-3055, 2005. 
[26] W. Sintunavarat and P. Kumam, "Common fixed point theorems for a pair of weakly compatible mappings in fuzzy metric spaces," Journal of Applied Mathematics, vol. 2011, Article ID 637958, 14 pages, 2011.

[27] W. Sintunavarat and P. Kumam, "Common fixed points for $R$ weakly commuting in fuzzy metric spaces," Annali dell'Universitá di Ferrara, vol. 58, no. 2, pp. 389-406, 2012.

[28] S. Chauhan, M. Imdad, and B. Samet, "Coincidence and common fixed point theorems in modified intuitionistic fuzzy metric spaces," Mathematical and Computer Modelling, vol. 58, Article ID 892900, 2013.

[29] S. Chauhan, B. D. Pant, and S. Radenović, "Common fixed point theorems for R-weakly commuting mappings with common limit in the range property," Journal of the Indian Mathematical Society. In press.

[30] M. Imdad, B. D. Pant, and S. Chauhan, "Fixed point theorems in Menger spaces using the $\left(C L R_{S T}\right)$ property and applications," Journal of Nonlinear Analysis and Optimization, vol. 3, no. 2, pp. 225-237, 2012.

[31] G. V. R. Babu and P. Subhashini, "Coupled common fixed point theorems of Ćirić type g-weak contractions with CLRg property," Journal of Nonlinear Analysis and Optimization, vol. 4, no. 1, 2013.

[32] S. Chauhan, M. A. Khan, and S. Kumar, "Unified fixed point theorems in fuzzy metric spaces via common limit range property," Journal of Inequalities and Applications, vol. 2013, 17 pages, 2013.

[33] S. Chauhan, M. A. Khan, and W. Sintunavarat, "Common fixed point theorems in fuzzy metric spaces satisfying $\phi$-contractive condition with common limit range property," Abstract and Applied Analysis, vol. 2013, Article ID 735217, 14 pages, 2013.

[34] M. Jain and S. Kumar, "A common fixed point theorem in fuzzy metric space using the property (CLRg)," Thai Journal of Mathematics. In press.

[35] M. Jain, K. Tas, S. Kumar, and N. Gupta, "Coupled fixed point theorems for a pair of weakly compatible maps along with CLRg property in fuzzy metric spaces," Journal of Applied Mathematics, vol. 2012, Article ID 961210, 13 pages, 2012.

[36] M. Kumar, P. Kumar, and S. Kumar, "Some common fixed point theorems using (CLRg) property in cone metric spaces," Advances in Fixed Point Theory, vol. 2, no. 3, pp. 340-356, 2012.

[37] R. K. Verma and H. K. Pathak, "Common fixed point theorems using property (E.A) in complex-valued metric spaces," Thai Journal of Mathematics, vol. 11, no. 2, 2013.

[38] G. Deschrijver, C. Cornelis, and E. E. Kerre, "On the representation of intuitionistic fuzzy t-norm and t-conorm," IEEE Transactions on Fuzzy Systems, vol. 12, pp. 45-61, 2004.

[39] G. Deschrijver and E. E. Kerre, "On the relationship between some extensions of fuzzy set theory," Fuzzy Sets and Systems, vol. 133, no. 2, pp. 227-235, 2003.

[40] M. Imdad, J. Ali, and M. Tanveer, "Coincidence and common fixed point theorems for nonlinear contractions in Menger PM spaces," Chaos, Solitons \& Fractals, vol. 42, no. 5, pp. 3121-3129, 2009.

[41] S. Chauhan, M. Imdad, and C. Vetro, "Unified metrical common fixed point theorems in 2-metric spaces via an implicit relation," Journal of Operators, vol. 2013, Article ID 186910, 11 pages, 2013.

[42] U. Gül and E. Karapınar, "On almost contraction in partially ordered metric spaces via implicit relation," Journal of Inequalities and Applications, vol. 2012, article 217, 2012.
[43] S. Gülyaz, E. Karapınar, and I. S. Yüce, "A coupled coincidence point theorem in partially ordered metric spaces with an implicit relation," Fixed Point Theory and Applications, vol. 2013, 11 pages, 2013.

[44] Nguyen Manh Hung, E. Karapınar, and Nguyen Van Luong, "Coupled coincidence point theorem in partially ordered metric spaces via implicit relation," Abstract and Applied Analysis, Article ID 796964, 14 pages, 2012.

[45] M. Imdad and S. Chauhan, "Employing common limit range property to prove unified metrical common fixed point theorems," International Journal of Analysis, vol. 2013, Article ID 763261, 10 pages, 2013. 


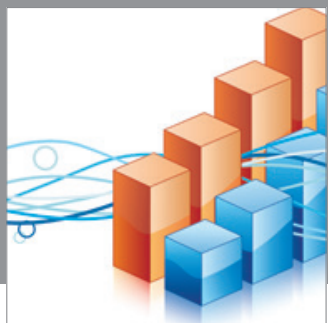

Advances in

Operations Research

mansans

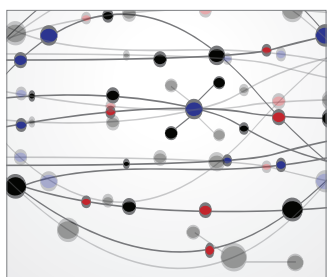

The Scientific World Journal
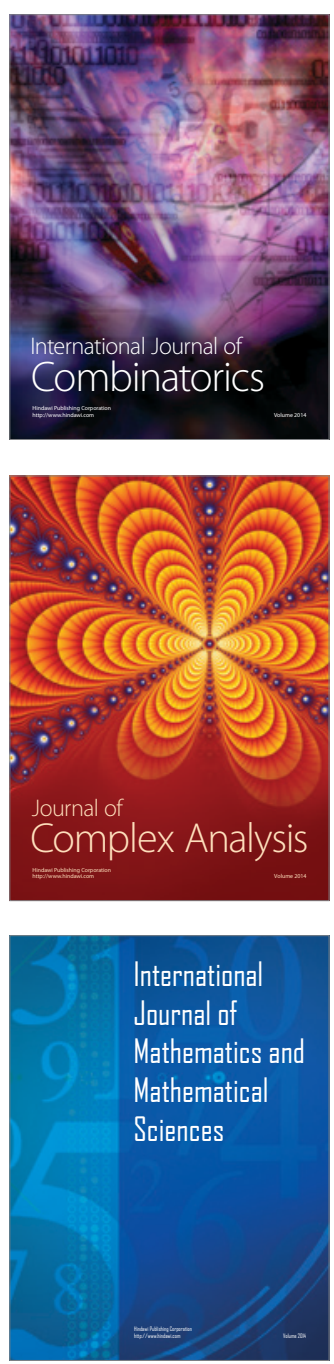
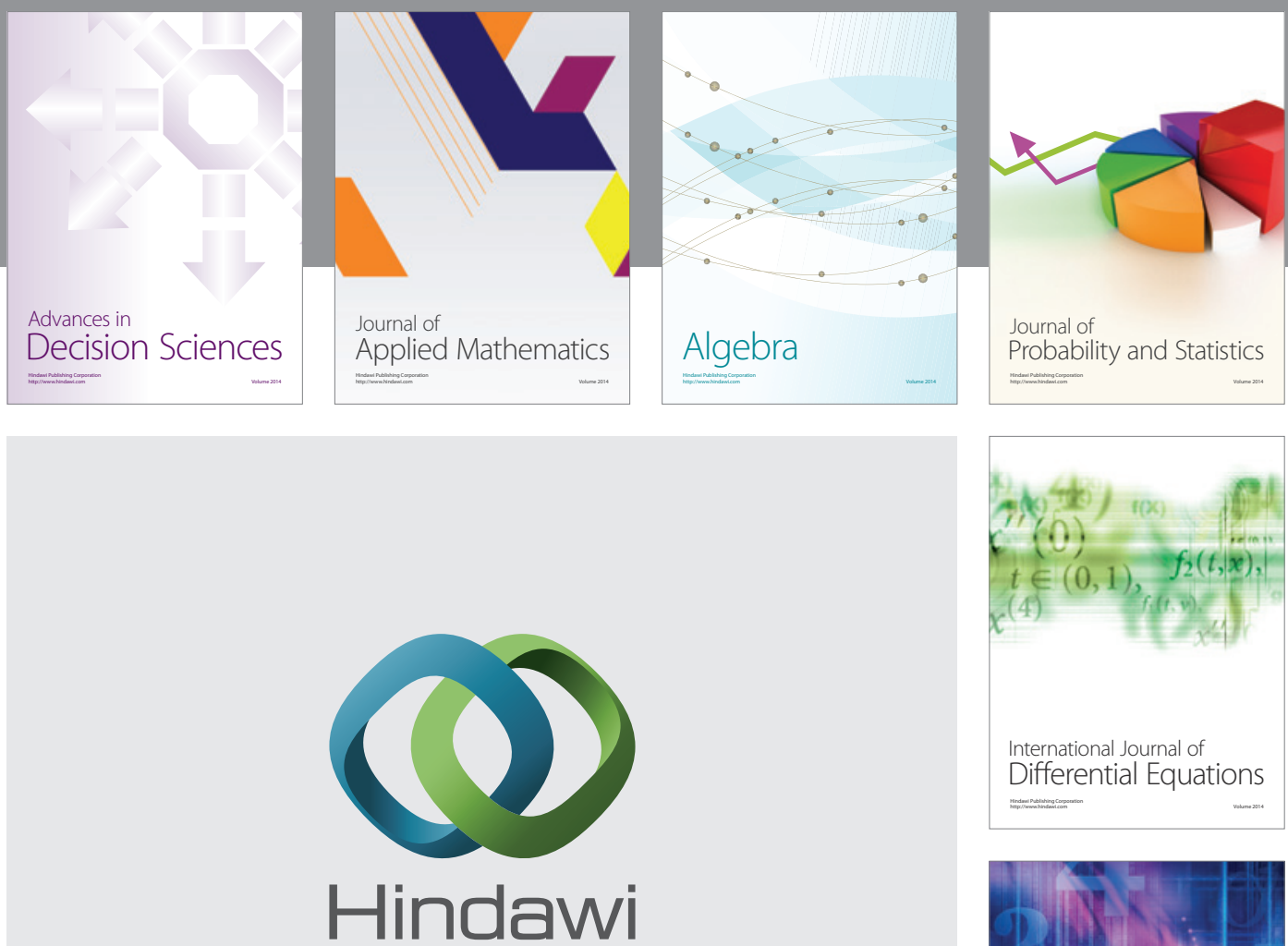

Submit your manuscripts at http://www.hindawi.com
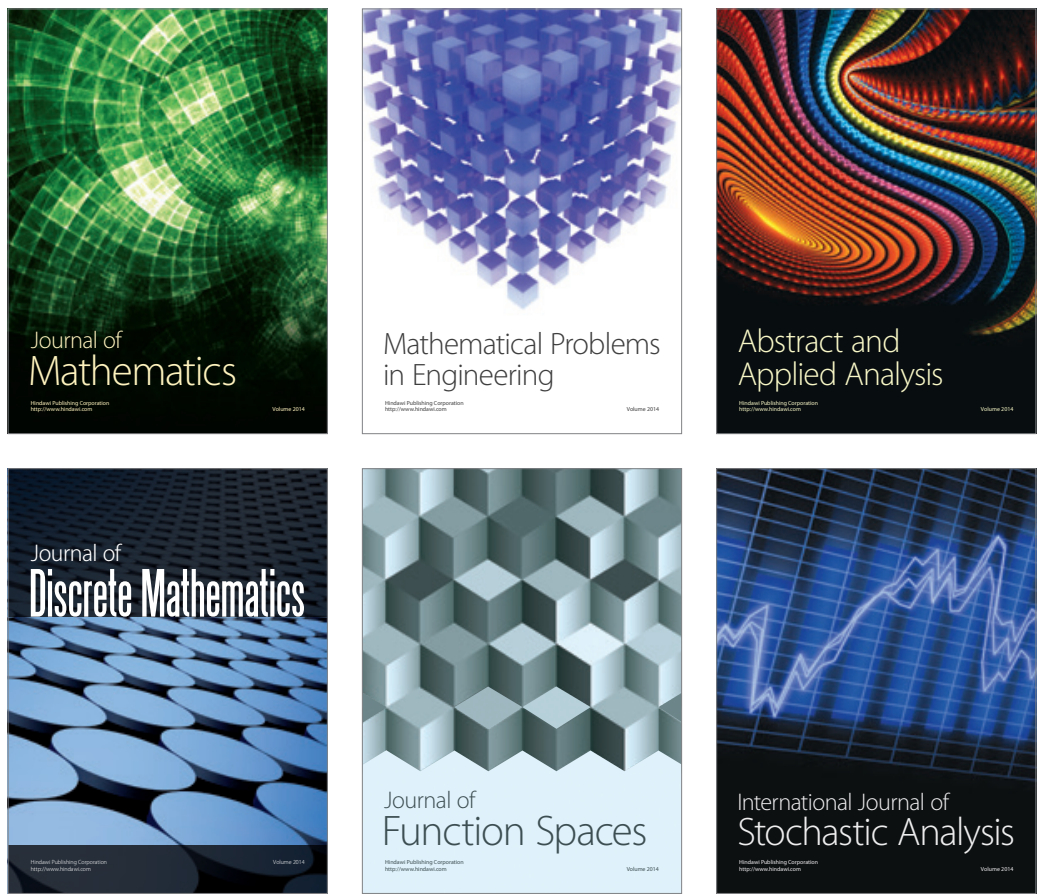

Journal of

Function Spaces

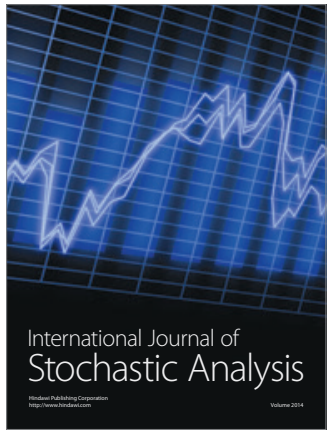

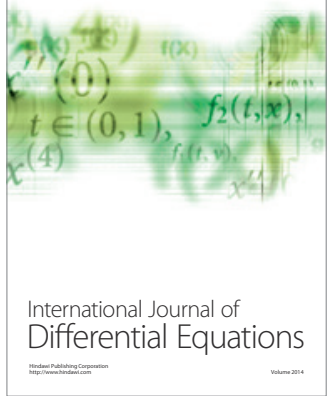
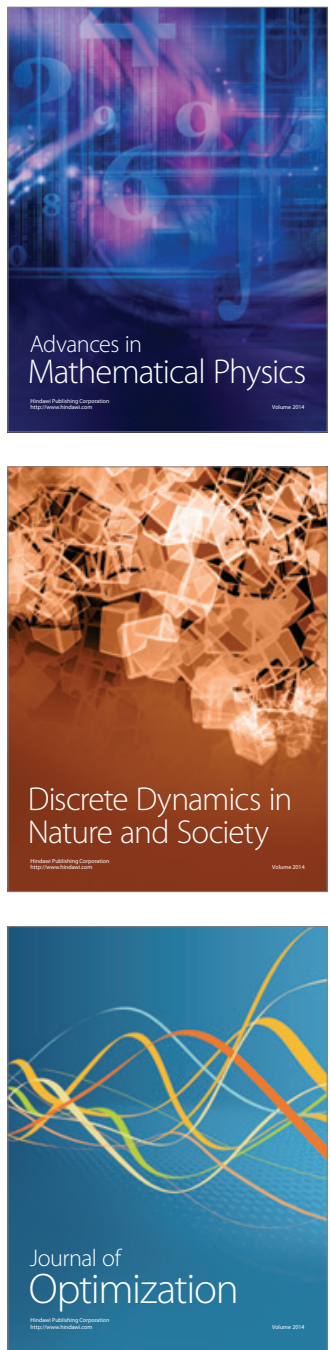